\title{
Changes in high intensity precipitation on the Northern Apennines (Italy) as revealed by multidisciplinary data over the last 9000 years
}

Stefano Segadelli ${ }^{1}$, Federico Grazzini ${ }^{2}$, Margherita Aguzzi ${ }^{2}$, Alessandro Chelli ${ }^{3}$, Veronica Rossi ${ }^{4}$, Maria T. De Nardo ${ }^{1}$, Roberto Francese ${ }^{3}$, Silvia Marvelli $^{5}$, Marco Marchesini ${ }^{5}$, Sandro $\mathrm{Nanni}^{2}$

${ }^{1}$ Geological, Seismic and Soil Service, Emilia-Romagna Region Administration, Bologna, Italy.

${ }^{2}$ Regional Agency for Prevention, Environment and Energy of Emilia-Romagna, Hydro-Meteo-Climate Service (ARPAE-SIMC), Bologna, Italy.

${ }^{3}$ Department of Chemistry, Life Sciences and Environmental Sustainability, University of Parma, Italy.

4 Department of Biological, Geological, and Environmental Sciences - BiGeA, Alma Mater Studiorum University of Bologna, Bologna, Italy.

5 Laboratory of Palynology and Archaeobotany - C.A.A. Giorgio Nicoli, San Giovanni in Persiceto (Bologna, Italy). 


\section{Supplementary Material}

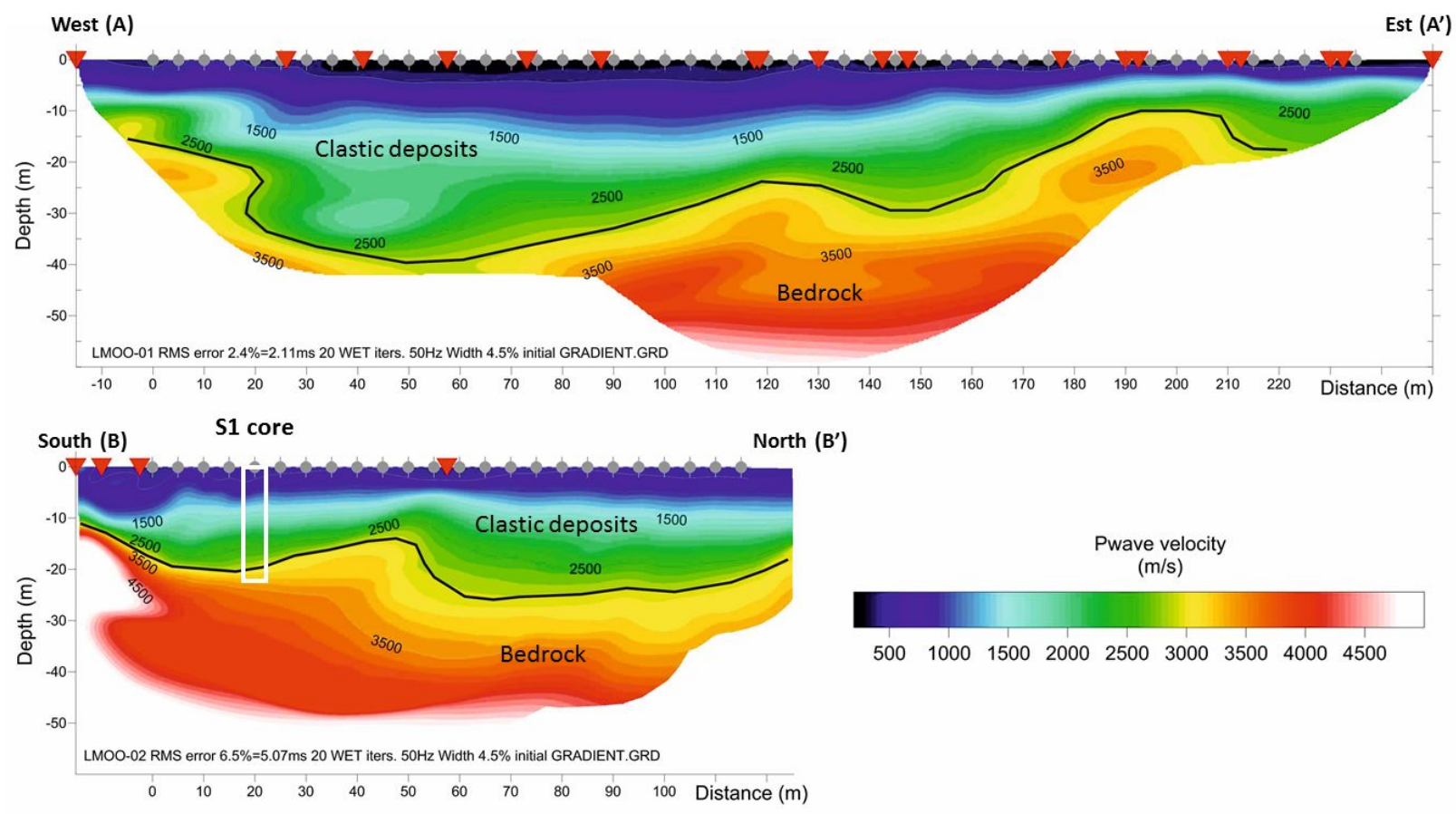

Figure SUP1. Seismic tomography of the underground lake Moo basin. For geophysical surveys tracks see Fig.2. 


\begin{tabular}{|c|c|c|c|c|c|}
\hline Lab. code & Core code & Core depth $(\mathrm{cm})$ & Material & $\begin{array}{c}\text { Age } \\
\text { (Cal year BP) }\end{array}$ & $\begin{array}{c}\text { Cal a AD } \\
( \pm 2 \text { sigma) }\end{array}$ \\
\hline LTL17681A & S1 & 113 & Organic matter & $82 \pm 45$ & 1680AD (29.4\%) 1764AD \\
\hline & & & (not determinable) & & $1801 \mathrm{AD}(66.0 \%) 1939 \mathrm{AD}$ \\
\hline LTL18275A & S2 & 237 & $\begin{array}{c}\text { Trunk grass } \\
\text { (not determinable) }\end{array}$ & $\begin{array}{c}178.69 \pm 0.55 \\
\mathrm{pMC}\end{array}$ & \\
\hline LTL18275Abis & S3 & $233-237$ & Organic matter (not determinable) & $\begin{array}{c}\text { It was not possible to } \\
\text { date, poor presence } \\
\text { of organic matter }\end{array}$ & \\
\hline LTL18274A & S4 & $279-281$ & Organic matter (not determinable) & $80 \pm 40$ & $\begin{array}{l}\text { 1681AD (26.5\%) 1737AD } \\
1804 \mathrm{AD}(68.9 \%) 1936 \mathrm{AD}\end{array}$ \\
\hline LTL18575A & S5 & $310-314$ & Small wood fragment (not determinable) & $102.12 \pm 0.50$ & \\
\hline LTL17682A & S6 & 455 & Pinus wood fragments & $3659 \pm 45$ & $\begin{array}{l}2194 \mathrm{BC}(2.2 \%) 2175 \mathrm{BC} \\
2144 \mathrm{BC}(93.2 \%) 1911 \mathrm{BC}\end{array}$ \\
\hline LTL18273A & S7 & $500-502$ & $\begin{array}{l}\text { Organic matter } \\
\text { (not determinable) }\end{array}$ & $4779 \pm 45$ & $\begin{array}{l}3649 \mathrm{BC}(85.7 \%) 3505 \mathrm{BC} \\
3427 \mathrm{BC}(9.7 \%) 3380 \mathrm{BC}\end{array}$ \\
\hline LTL18277A & S8 & 760 & $\begin{array}{l}\text { Organic matter and small wood fragment } \\
\text { (not determinable) }\end{array}$ & $5686 \pm 45$ & $\begin{array}{l}4683 B C(6.0 \%) 4631 B C \\
4623 B C(87.6 \%) 4446 B C \\
4418 B C(1.8 \%) 4400 B C\end{array}$ \\
\hline LTL18278A & S9 & $860-865$ & Abies alba wood fragment & $6065 \pm 45$ & $\begin{array}{l}5205 \mathrm{BC}(4.1 \%) 5165 \mathrm{BC} \\
5077 \mathrm{BC}(91.3 \%) 4835 \mathrm{BC}\end{array}$ \\
\hline LTL18577A & S11 & 949 & Organic matter (not determinable) & $6363 \pm 45$ & $5470 \mathrm{BC}(88.8 \%) 5291 \mathrm{BC}$ \\
\hline LTL18272A & S12 & 959 & Organic matter (not determinable) & $\begin{array}{c}159.33 \pm 0.48 \\
\text { pMC }\end{array}$ & \\
\hline LTL17683A & $\mathrm{S} 13$ & 1044 & $\begin{array}{l}\text { Plant fragments carboniosi (not } \\
\text { determinable) }\end{array}$ & $8600 \pm 45$ & $7722 \mathrm{BC}(95.4 \%) 7551 \mathrm{BC}$ \\
\hline
\end{tabular}

Table TS2 Full list of Radiocarbon sample age and description details. 